International Journal of Engineering \&Technology, $7(1.8)(2018) 130-138$
International Journal of Engineering \& Technology
SPC
Website: www.sciencepubco.com/index.php/IJET
Research paper

\title{
Alleviation of VAr Impact on Critical Loading Margin with Redispatch in Deregulated Power Systems
}

\author{
Venkateswarlu, A.N ${ }^{1 *}$, Tulasi Ram, S.S ${ }^{2}$, Sangameswara Raju.P ${ }^{3}$ \\ ${ }^{I}$ Department of Electrical and Electronics Engineering, Vignan's Lara Institute of Technology and Science, \\ Vadlamudi, Guntur, A.P., India \\ ${ }^{2}$ Department of Electrical and Electronics Engineering, JNTUH College of Engineering, Hyderabad, Telangana, India \\ ${ }^{3}$ Department of Electrical and Electronics Engineering, SVU College of Engineering, Tirupati, A.P., India \\ *Email: atyam2000@gmail.com
}

\begin{abstract}
The stability management under deregulated environment has become typical task to the system due to random nature of load pattern and generation schedules. In addition, the regular uncertainties in system operation like line outage, generator outage or change in loading level are also causing to change in stability as well as security margins significantly. In order to manage transmission system security, the system operator can go for redispatch as a short term solution. In this article, an attempt is made to clear reactive power loading (VAr) impact on voltage instability margin or Critical Loading Margin (CLM). An Interior Point -Optimal Power Flow (IP-OPF) is applied to make system secured under ( $\mathrm{N}-1)$ line contingencies. Using this secured schedule, the CLM is computed using Continuous Power Flow $(\mathrm{CPF})$ for the two operating scenarios i.e., without VAr and with VAr loading on the system. The case study is simulated on IEEE 14-bus test network and outcome is validating that, the redispatch can also be apt for CLM enhancement even under contingencies as short term solution for stability management in real time.
\end{abstract}

Keywords: Critical Loading Margin; Continuous Power Flow; Deregulation; Interior Point-Optimal Power Flow and Redispatch.

\section{Introduction}

Power network stability is a complex issue that has been challenging power system engineers for a lot of years. The various stability problems and their fundamental explanation can be found in [1]. One of such stability problems, voltage stability is highly related with reactive power control in the system. Reactive power management through voltage control is the key aspect of efficient and reliable power system operation. The basic objectives of reactive power management are as follows [1]: (i) to maintain all bus voltages within acceptable limits (ii) to enhance system stability by maximum utilization of the transmission system, and (iii) to minimize reactive power flow in transmission lines and consequently to achieve minimum transmission losses. All these objectives ensure that the transmission network operates efficiently by having a capability to transfer more active power. In the current scenario, this helps to improve competition in open access transmission.

In the history of power network control and operation, reactive power plays a vital role to maintain security as well as stability. The insufficient reactive power reserve is one of the major causes for various power system blackouts. A few of them have been reported with complete analysis of different causes for these failures in the literature. Cost-effective limitations with reorganized atmosphere, involuntary action of protecting schemes, non effective design of traditional load-flaking approach and inadequate reactive power sources are a few of the major causes initiating these blackouts. Amongst the entire causes, deficit of reactive power support is a chief noteworthy aspect for voltage instability. So as to preserve voltage for network consistency, equally reactive and active power consumptions should be controlled. Nevertheless, a straight linkage between reactive power and voltage will make it feasible to control voltage to the preferred values by controlling the reactive power [2].

In common, the load contains both the reactive and active components. The transmission of power in AC networks involves the reactive components. Generation of reactive power is also depending on the reactive compensation/reserve in the network. Therefore, it is vital to control and supervise the reactive power consuming elements and the reactive power resources (sink) to preserve proper voltages in grid within secure, safe and specified limits. Thus control of reactive power forms the chief apprehension of bulk power grids.

The generation of reactive power is chiefly depending on the control of voltage i.e., controlling devices at the distribution side, excitation at the generation side and compensation at the transmission side. Minimum reactive power yield from a generator can be achieved either by the integration of reactive power compensation devices at load side or proper excitation control. Usually, the object of reactive power (VAr) planning problem is to present a smallest number of latest reactive power supplies to promise only voltage viability constraints in post-contingency and 
regular state. Different researches are carried out for this theme [34]. Work is as well done on best possible reactive power planning approach against voltage collapse in [5]. By using OPF, it is possible to adjust the generator bus voltages to lessen reactive power output and reduced operating cost can be obtained consequently [6].

This work is intended to clear reactive power impact on voltage stability and it can be improved by using redispatch under $(\mathrm{N}-1)$ line contingencies. This paper has been structured as follows: after introduction, the methodology has been explained in brief in section 2. In section 3, the redispatch using IP-OPF has been explained and in Section 4, the CLM assessment by using CPF is given. In section 5 , the results along with discussions and in section 6 , the conclusion are imparted.

\section{Methodology}

The primary goal of Independent System Operator (ISO) in deregulated power system is to lessen the cost of meeting load demand for power network whilst maintain the safety of network. The cost related with the power network may depend on the circumstances, but in common, they can be attributed to the cost of power generation (megawatts) at every generator. In general, the maintenance of system safety would necessitate keeping every device in power network in its preferred operating range at steadystate. This will include upper limit and lower limit of outputs of generators, highest MVA flow in transmission lines and transformers, as well as keeping the network bus voltages in specific range.

In general, the generation schedule in deregulation is happening based on the electricity market participants offer bids only for real power injections and withdrawals. But in real time scheduling, the system operator has to consider the reactive power also in to account to maintain security. Since reactive power consumption in transmission network is not likely to estimate before the dispatch, the reactive power management has become one of the typical jobs and it is also a significant action in the power system control and operation. Unluckily, reactive power has become one of the

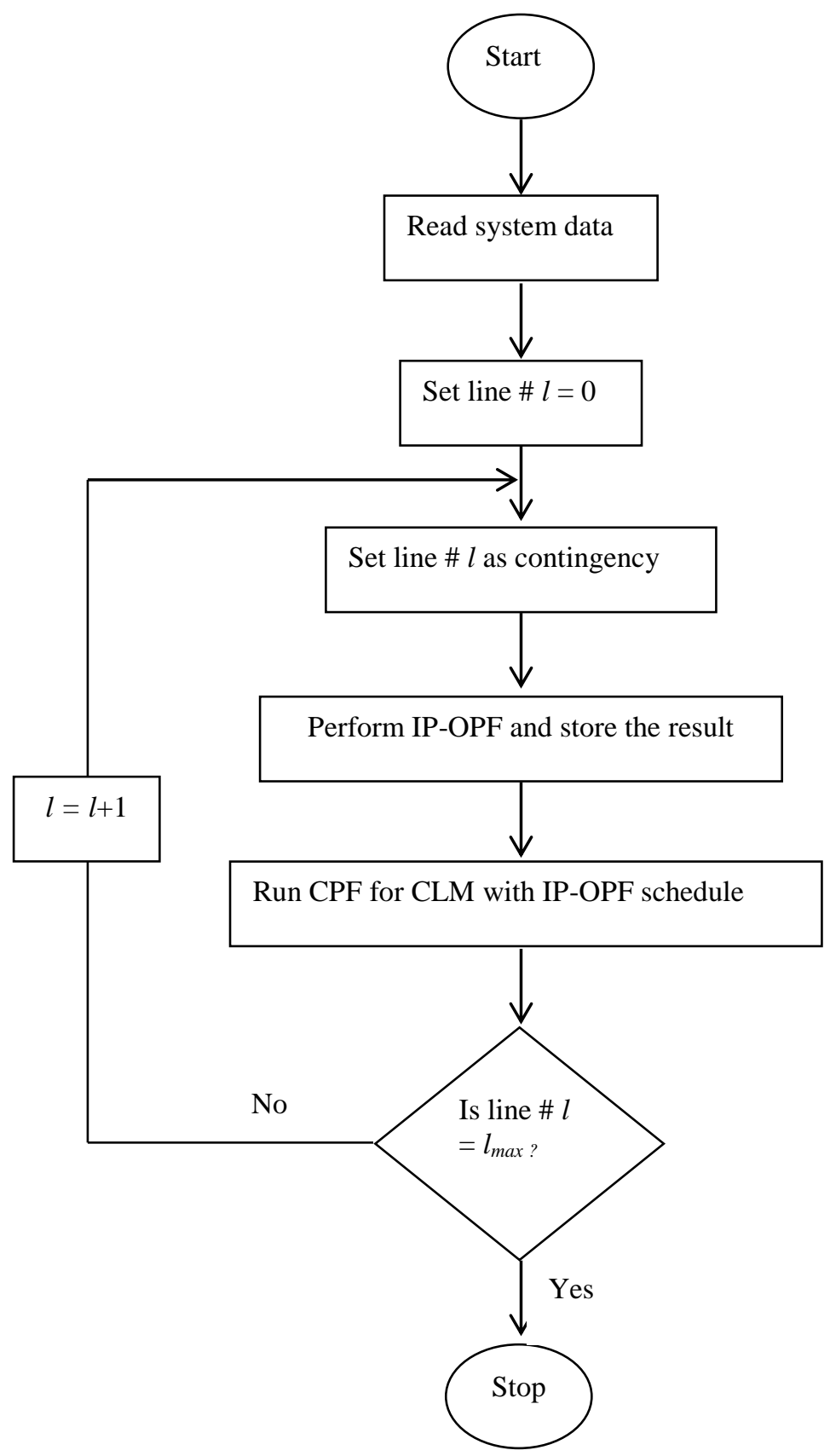

Fig 1: Flow chart for security constrained critical loading margin assessment 
main auxiliary services to procure from its service provider in the majority of electricity markets and it is required to minimize the procurement of reactive power from VAr service providers by extracting added support from supporting/ controlling devices. The producers of reactive power in the system can be mostly either FACTS devices, generators or synchronous condensers. By extracting best possible yield from VAr supporting/controlling devices, it is possible to get less reactive power yield from generators and results in decreased cost of reactive power.

In this work, IP-OPF is run for the stand case, i.e., pre-disturbance case and the line flows and voltage profile of the system are noted By considering this operating condition as input to the CPF, the critical loading margin is obtained. The process is repeated for the whole $(\mathrm{N}-1)$ line contingency with and without the consideration of VAr loading on the network. The complete procedure has been given as a flow chart in the Fig. 1.

\section{Redispatch using IP-OPF}

The feasibility of the bottom case market program will depend upon on system support. Transmission congestion in the deregulated atmosphere refers to 'lack of ability of transmission system to hold up competitive electricity market driven schedule'. The possibility of transmission system's incapability would occur in market schedule because of either overcapacity of transformers, transmission lines or due to defiance of any other operational constraint. In sequential electricity market, the program settle merely depend on financially viable grounds only at initial step for energy equilibrium and afterward, it will regulate according to the operational constraint for network secured operation at next stair. However, in concurrent electricity market agreement, both security constraints and energy balance will be considered in amalgamation.

The remedial/defensive actions are necessary to dispatch market schedule with the acceptable operational constraints of network will be called as congestion management $(\mathrm{CM})$ approach. The chief object of congestion management approach is communal benefit maximization and therefore the CM approach will wander for circumstance to circumstance or market to market. A general structure for congestion release is developed to withstand under usual plus unusual conditions too. Depending on the price to be incurred with these events, the CM approach can be divided into two large categories i.e., cost-free and non-cost-free approaches. Likewise, they can as well be divided into technical and nontechnical categories. Non-technical approaches will again be categorized into market oriented and non-market oriented approaches. The complete investigation of a variety of approaches can be found in the literature [7].

The transmission system security assurance generation program can be obtained using IP-OPF method. Under the lack of network support to drive market schedule, Interior Point-Optimal Power Flow (IP-OPF) [8] has been implemented not only to minimize redispatch /congestion cost but also to control reactive power cost subjected to technical benefits like loss minimization and voltage profile improvement.

\section{CLM assessment by using CPF}

The continuation power flow (CPF) study is tough, flexible and suitable to solve power flow problems with convergence difficulty. Nevertheless, this method will consume time and awfully slow. Therefore, best approach is to utilize combination of continuation method and traditional power flow (CPF) method i.e., FD or NR method. Beginning from bottom case, power flow is solved by means of a traditional method to calculate power flow solutions for consecutively rising load levels till a solution cannot be obtained. After this, the continuation method is restored to attain power flow solution. Generally, the continuation method is needed merely if solutions are necessary precisely at and past the critical point [9]. The complete information regarding CPF can be found in $[10,11]$.

Maximum Loading Capability (MLC) is a gauge for Voltage Stability Margin (VSM) of the network. This can also be called as Critical Loading Margin (CLM). In general, voltage stability analysis using Continuous Power Flow (CPF) gives the value of MLC of the network. For a small increment of load beyond this margin can cause to voltage instability/voltage collapse. Therefore, the power network should have the significant margin from working point to voltage collapse point for voltage security. Voltage stability and voltage security are equally greatly depend on reactive and active power sources of the power network and chiefly reactive resources are most efficient [12].

$\mathrm{CPF}$ is the method to find out a variety of power flow solutions opening at some bottom load and leading to steady-state voltage stability limit (critical point) of network [13]. The convergence of traditional continuation power flow computation can't be assured near collapse point due to the singularity of the Jacobean matrix. To prevail over it, locally parameterized continuation power flow method has been proposed in $[14,15]$. The enhanced method can successfully promise the convergence of continuation power flow computation still near collapse point.

The basic mathematic equations involved in CPF method are given here [13]. The net reactive and active power injections at source and sink buses are the functions of lambda $(\lambda)$ and will be given by

$$
P_{i}=P_{i 0}+\lambda L_{P i}
$$

$Q_{i}=Q_{i 0}+\lambda L_{Q i}$

where

$\lambda \quad$ : is parameter controlling the amount of power

injection

$P_{i 0} \quad:$ is bottom case real power injection at i th bus

$Q_{i 0} \quad:$ is bottom case reactive power injection at i th bus

$L_{P i} \quad:$ is real power load participation factors at $i$ th bus

$L_{Q i} \quad:$ is reactive power load participation factors at $i$ th

Bus

The conventional power flow equations are augmented by an additional equation for lambda $(\lambda)$ and are expressed as

$f(\theta, V, \lambda)=0$

where

$V \quad:$ is the vector of bus voltage magnitudes

$\theta \quad:$ is the vector of bus voltage angles

Once a bottom case (for $\lambda=0$ ) solution is established, the subsequent solution can be predicted by assuming a suitably sized step in a path of the tangent to solution pathway. Tangent vector can be obtained as given below:

$d[f(\theta, V, \lambda)]=f_{\theta} d \theta+f_{V} d V+f_{\lambda} d \lambda$ 
Because equation (4) is short of rank, a random value like 1 (one) is assigned as one of elements of tangent vector $t=[d \theta, d V, d \lambda]^{T}= \pm 1$, i.e., $t_{k}= \pm 1$. Therefore,

$\left[\begin{array}{lll}f_{\theta} & f_{V} & f_{\lambda} \\ & e_{k} & \end{array}\right][t]=\left[\begin{array}{c}0 \\ \pm 1\end{array}\right]$

where $e_{k}$ is the row vector with every element equal to zero, except for $\mathrm{k}^{\text {th }}$ entry, which will be to 1 (one). The latest solution following perturbation can now be calculated as below:

$$
\left[\begin{array}{c}
\theta^{*} \\
V^{*} \\
\lambda^{*}
\end{array}\right]=\left[\begin{array}{l}
\theta \\
V \\
\lambda
\end{array}\right]+\varepsilon\left[\begin{array}{l}
d \theta \\
d V \\
d \lambda
\end{array}\right]
$$

where $\varepsilon$ is a scalar used for altering the step size.

The latest solution obtained in the Eqn. (6) may disobey the given limits. Hence, it is required to correct continuation parameter. Corrector is somewhat adapted in Newton power flow algorithm in which Jacobean matrix has been augmented by an equation to consider for continuation parameter.

Let $x=[\theta, V, \lambda]^{T}, x_{k}=\eta$, be the latest set of equations which will take up the form:

$$
\left[\begin{array}{c}
f(x) \\
e_{k}-\eta
\end{array}\right]=[0]
$$

\section{Results and discussions}

This case study is performed to clear the reactive power load on system voltage stability. Voltage stability is measured in terms of critical loading margin (CLM), i.e., nose point of PV curve for various levels of reactive power on the system. The simulation studies have been performed on modified IEEE 14-bus test network. The test system contains 17 transmission lines, 5 generator buses and 3 transmission lines with regulating transformers. The system has $259 \mathrm{MW}+j 73.5 \mathrm{MVAr}$ load. The details can be found in [16].

\subsection{Impact of Reactive Power Load on Critical Loading Margin}

Primarily, the reactive power load on the system at each bus is made equal to zero and corresponding CLM is obtained using CPF method. The CLM for 0\% reactive power load is equal to 2.267 p.u. Later, the percentage of reactive load is increased by $10 \%$ in steps and corresponding CLM values are measured. The decrement in CLM as reactive power level increment can be observed in Table 1. For the \%100 reactive power load (it is the standard load of the system), the CLM is equal to 2.006 and this is around $11.51 \%$ decrement to the case 'without reactive power load'. The PV curves for different reactive power loading levels are given in Fig. 2(a) to Fig. 2(k).

Table 1: CLM w.r.t. percentage of reactive load on system

\begin{tabular}{ccccc}
\hline $\begin{array}{c}\text { \% Reactive } \\
\text { Power load }\end{array}$ & CLM (p.u) & $\begin{array}{c}\text { \% Reactive } \\
\text { Power load }\end{array}$ & CLM (p.u) & $\begin{array}{c}\text { \% Reactive } \\
\text { Power load }\end{array}$ \\
\hline 0 & 2.267 & 40 & 2.187 & 80 \\
10 & 2.251 & 50 & 2.171 & 90 \\
20 & 2.234 & 60 & 2.145 & 100 \\
30 & 2.214 & 70 & 2.115 & 2.046 \\
\end{tabular}

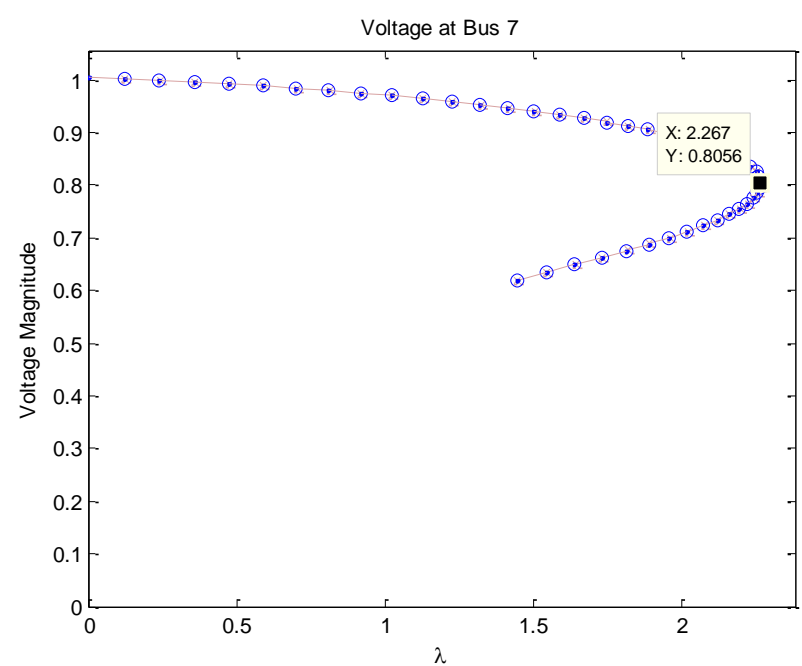

Fig 2(a): PV Curve with $Q=0 \%$

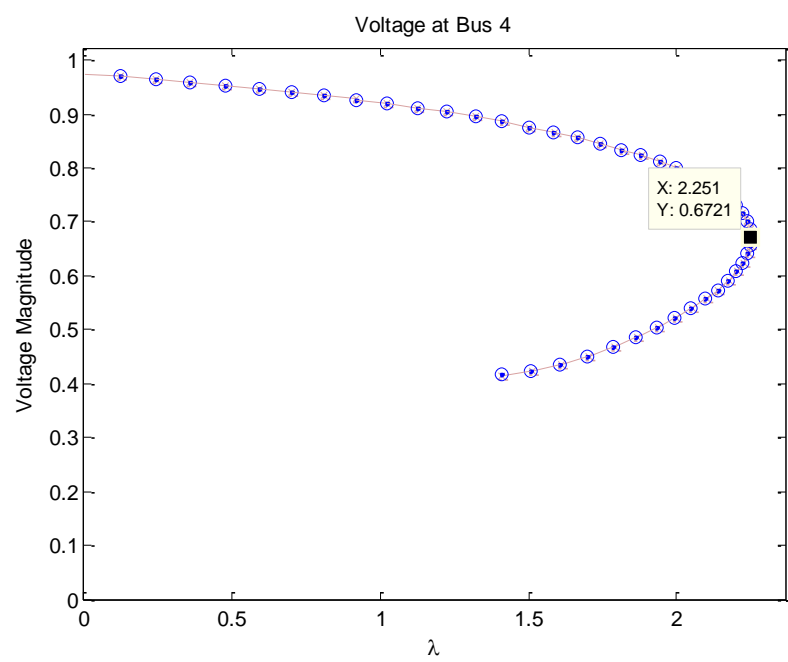

Fig 2(b): PV Curve with $Q=10 \%$ 


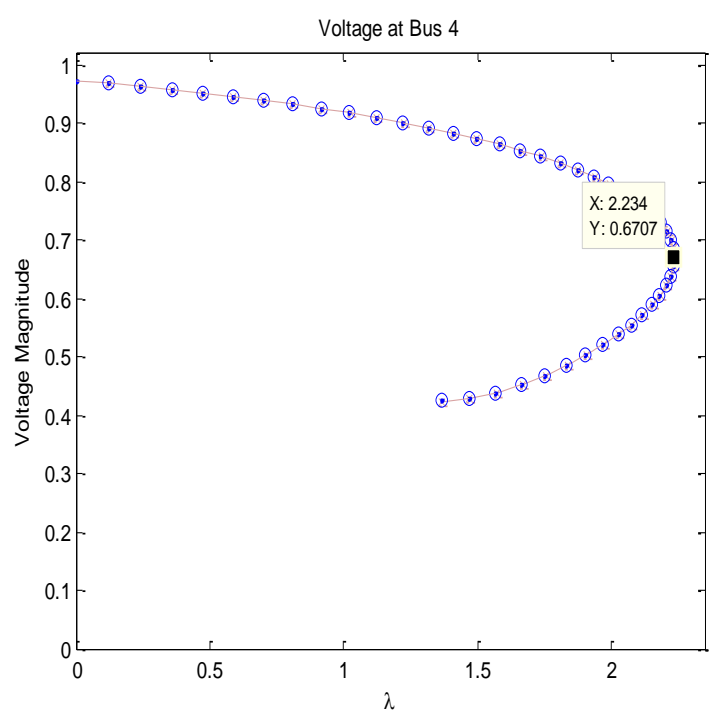

Fig. 2(c): PV Curve with $Q=20 \%$

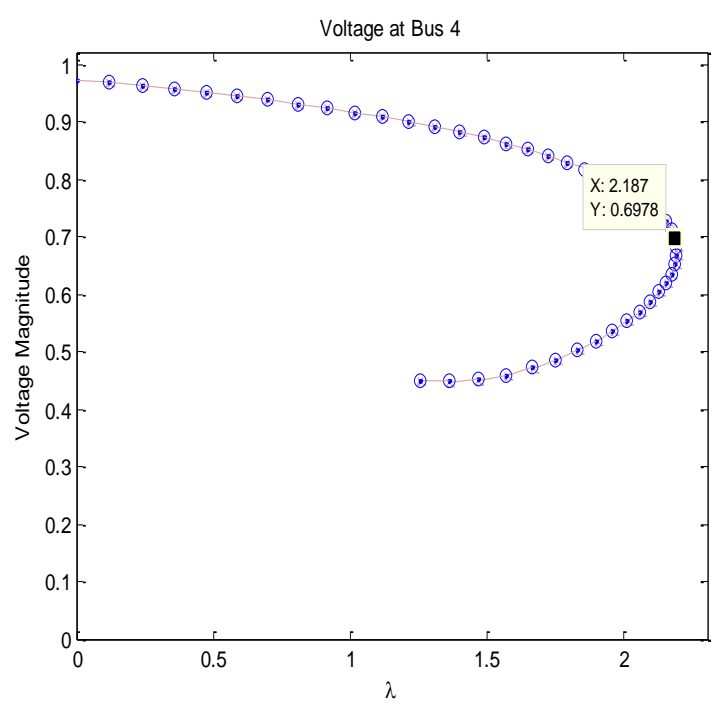

Fig. 2(e): PV Curve with $Q=40 \%$

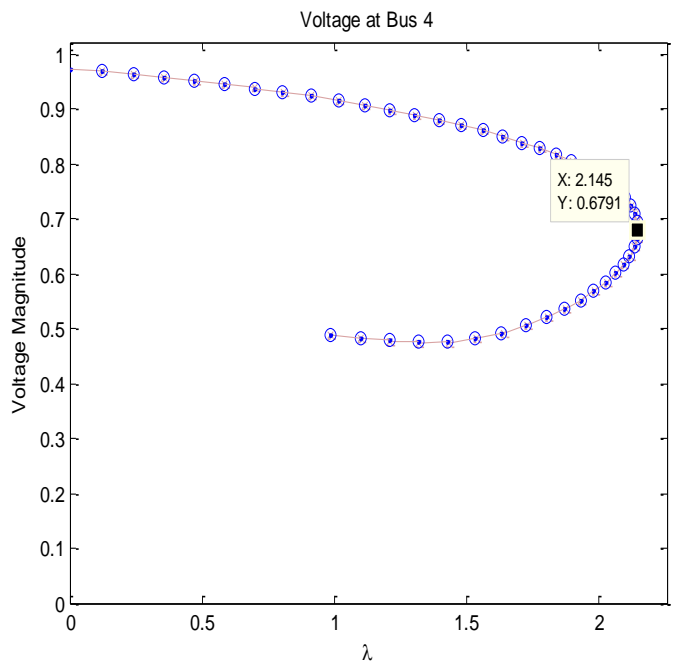

Fig 2(g): PV Curve with $Q=60 \%$

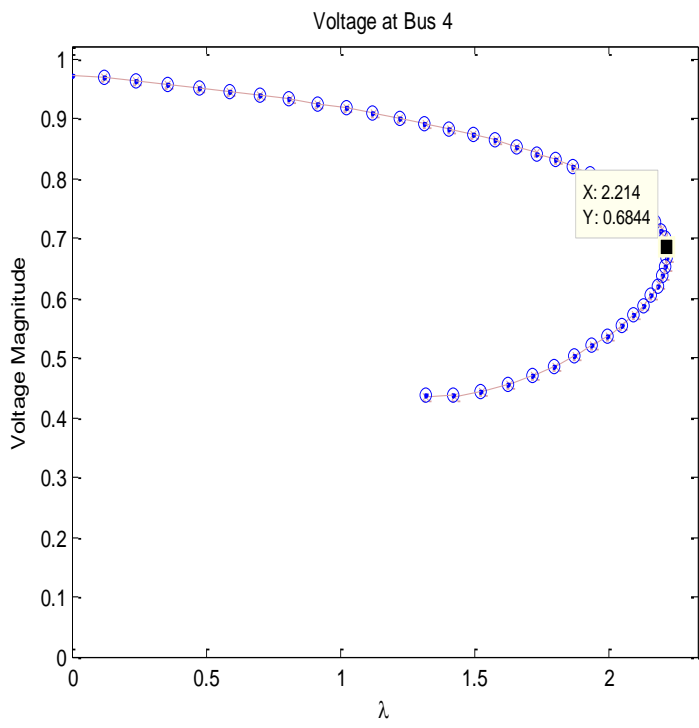

Fig 2(d): PV Curve with $Q=30 \%$

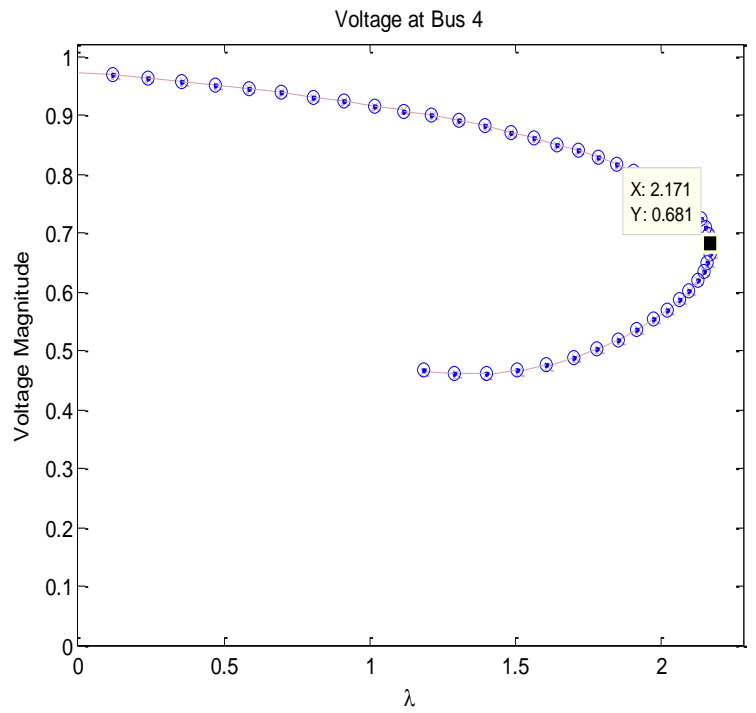

Fig 2(f): PV Curve with $Q=50 \%$

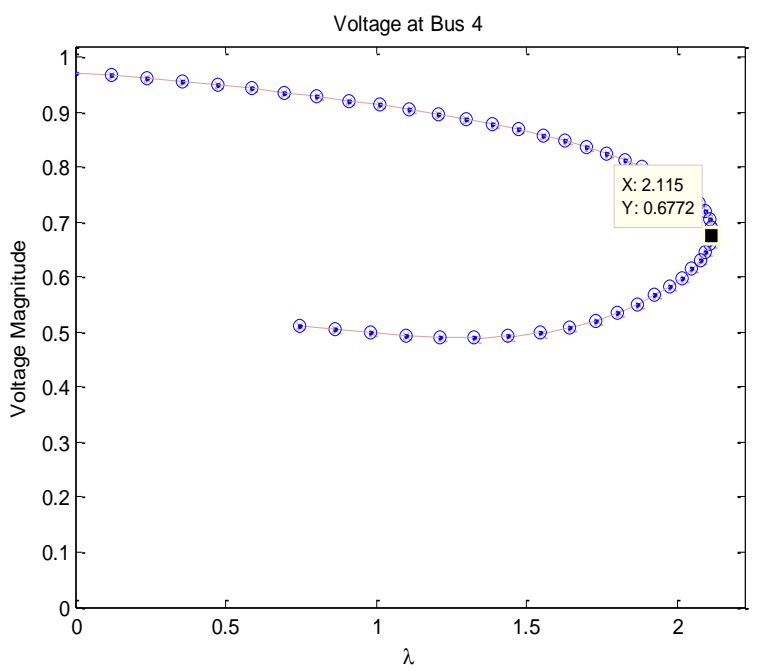

Fig 2(h): PV Curve with $Q=70 \%$ 


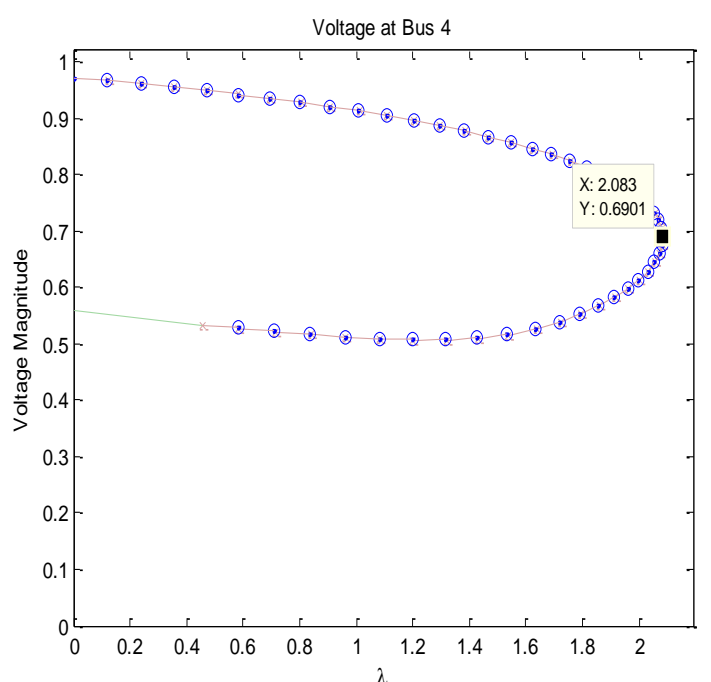

Fig 2(i): $P V$ Curve with $Q=80 \%$

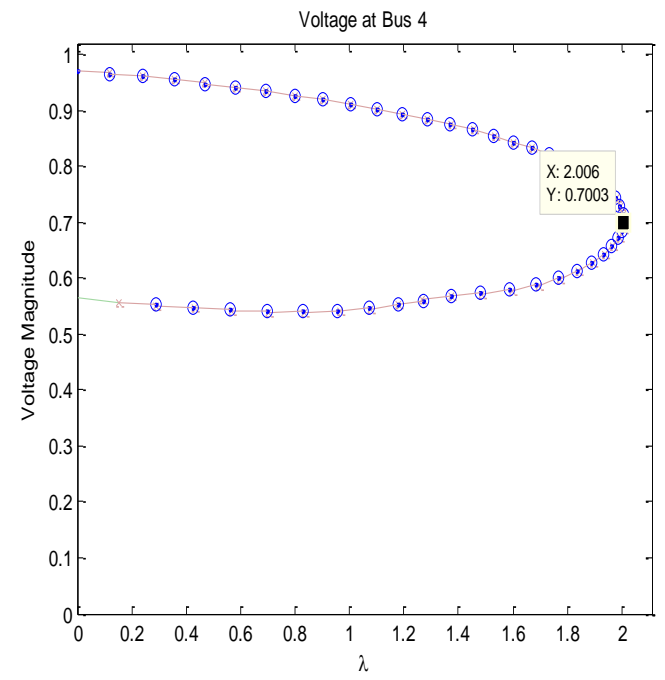

Fig 2(k): PV Curve with $Q=100 \%$

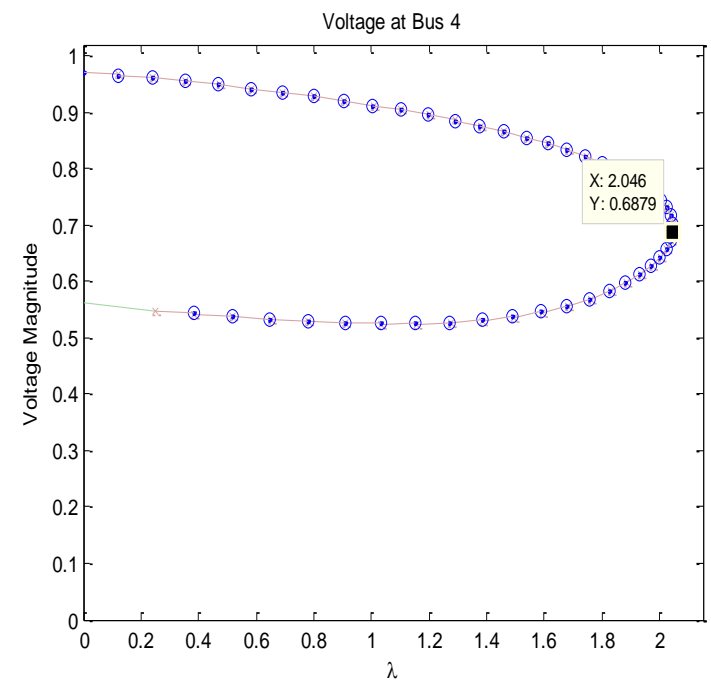

Fig 2(j): PV Curve with $Q=90 \%$

Fig 2: PV curves for different VAr loading conditions

\subsection{Critical Loading Margin with Base Case Schedule under (N-1) Line Contingencies}

The case studies are divided in to two categories i.e., (i) without reactive load on the system and (ii) with reactive load on system. Primarily, the test system reactive load data is modified to zero at all the buses. Since the reactive power load is assumed as zero in this case, the drop is due to reactive nature of the transmission system.

The AC-OPF is run for the base case that is the pre-disturbance case and the voltage profile and line flows of the system are noted.
By considering this operating condition as input to the CPF, the critical loading margin is obtained. The procedure is repeated for all (N-1) line contingency cases and the results have been tabulated and given in the Table 2. Furthermore, PV curves at critical bus under line (1-5) and (12-13) are given in Fig. 3 and Fig. 4 for the two scenarios. The similar curves for other line contingencies are not given because of space constraint.

Table 2: Critical loading margin for different scenarios

\begin{tabular}{|c|c|c|c|c|c|}
\hline Line outage & Without Q & With Q & Line outage & Without Q & With Q \\
\hline Base case & 2.267 & 2.006 & $5-6$ & 1.017 & 0.772 \\
\hline $1-2$ & 0.287 & 0.279 & $6-11$ & 2.179 & 1.562 \\
\hline $1-5$ & 1.972 & 1.778 & $6-12$ & 2.23 & 1.936 \\
\hline $2-4$ & 1.676 & 1.571 & $7-9$ & 1.660 & 1.092 \\
\hline $2-5$ & 1.778 & 1.636 & $9-10$ & 2.220 & 1.962 \\
\hline $3-4$ & 2.064 & 1.833 & $9-14$ & 2.083 & 1.480 \\
\hline $4-5$ & 2.208 & 2.021 & $10-11$ & 2.221 & 1.717 \\
\hline $4-7$ & 1.979 & 1.751 & $12-13$ & 2.264 & 1.990 \\
\hline $4-9$ & 2.192 & 1.886 & $13-14$ & 2.034 & 1.353 \\
\hline
\end{tabular}




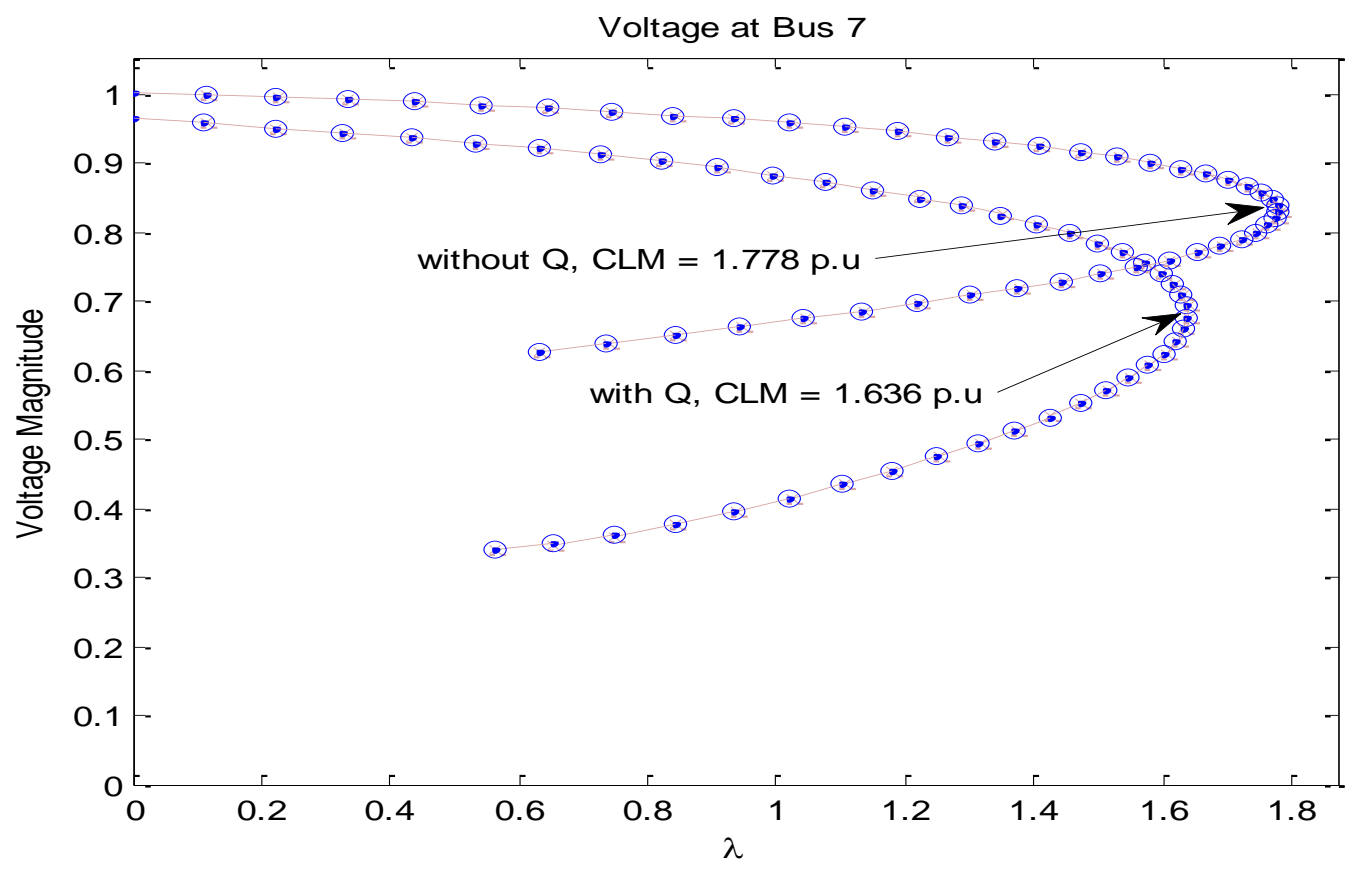

Fig 3: CLM under line (1-5) contingency without and with VAr loading

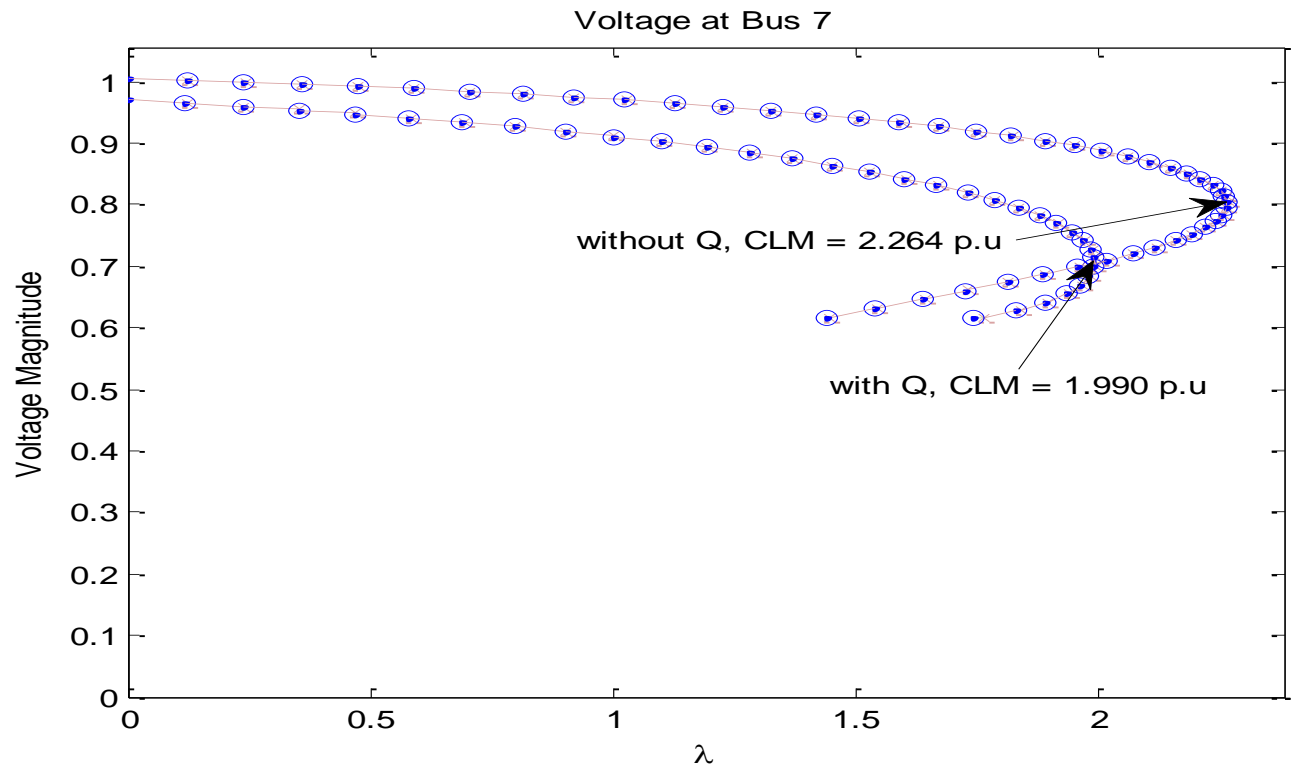

Fig 4: CLM under line (12-13) contingency without and with VAr loading

\subsection{Critical Loading Margin with Re-Schedule under (N-1) Line Contingency}

As highlighted in the Sections 5.1 and 5.2, both the reactive power loading as well as line contingencies are affecting the CLM of the system. In this section, an attempt is made to clear the redespatch impact under respective (N-1) contingency while assessing the CLM of the system.

The generation schedule for all the cases are obtained using DCOPF [16] method for the case of without reactive power loading on system. Similarly for the case of reactive power loading on system, AC-OPF [16] method is used to obtain generation schedule. The schedules for without reactive load and with reactive load are given in Table 3 . The CPF is simulated under $(\mathrm{N}$ 1) line contingency with its respective re-schedule as given in Table 4. The improved CLM with market reschedule can be observed in Table 4 as compared with Table 2.

In short, the impact of reactive power loading on CLM under various $(\mathrm{N}-1)$ line contingences is given in Fig. 5 for the two cases. Similarly, the impact of reschedule without VAr loading and with $\mathrm{VAr}$ loading on CLM under various contingences is illustrated in Fig. 6 and Fig. 7 respectively. 
Table 3: Security constrained schedule in (MW) under $(\mathrm{N}-1)$ line contingencies

\begin{tabular}{|c|c|c|c|c|c|c|c|c|c|c|}
\hline \multirow{2}{*}{ Outage } & \multicolumn{5}{|c|}{ without VAr load } & \multicolumn{5}{|c|}{ with VAr load } \\
\hline & PG1 & PG2 & PG3 & PG4 & PG5 & PG1 & PG2 & PG3 & PG4 & PG5 \\
\hline Base & 220.97 & 38.03 & - & - & - & 194.33 & 36.72 & 28.74 & - & 8.50 \\
\hline $1-2$ & 110.00 & 41.43 & 35.86 & 35.86 & 35.86 & 109.87 & 41.01 & 65.06 & 13.26 & 37.05 \\
\hline $1-5$ & 200.00 & 40.25 & 6.25 & 6.25 & 6.25 & 178.94 & 35.58 & 24.25 & 10.73 & 21.62 \\
\hline $2-3$ & 183.26 & 31.54 & 44.20 & - & - & 177.71 & 33.12 & 58.33 & - & - \\
\hline $2-4$ & 220.97 & 38.03 & - & - & - & 190.31 & 35.63 & 23.23 & - & 20.07 \\
\hline $2-5$ & 220.97 & 38.03 & - & - & - & 192.56 & 36.07 & 24.79 & 1.45 & 13.98 \\
\hline $3-4$ & 220.97 & 38.03 & - & - & - & 194.22 & 36.73 & 31.63 & - & 5.66 \\
\hline $4-7$ & 220.97 & 38.03 & - & - & - & 192.62 & 36.39 & 23.91 & - & 15.33 \\
\hline $4-9$ & 220.97 & 38.03 & - & - & - & 193.89 & 36.63 & 27.37 & - & 10.39 \\
\hline $5-6$ & 214.93 & 36.99 & - & - & 7.08 & 188.35 & 35.68 & 16.13 & 28.47 & - \\
\hline $6-11$ & 220.97 & 38.03 & - & - & - & 193.78 & 36.62 & 27.76 & - & 10.20 \\
\hline $6-12$ & 220.97 & 38.03 & - & - & - & 194.26 & 36.71 & 28.64 & - & 8.90 \\
\hline $6-13$ & 220.97 & 38.03 & - & - & - & 193.55 & 36.59 & 27.37 & - & 11.58 \\
\hline $7-9$ & 220.97 & 38.03 & - & - & - & 194.57 & 36.75 & 28.88 & - & 8.46 \\
\hline $9-10$ & 220.97 & 38.03 & - & - & - & 194.86 & 36.81 & 29.58 & - & 7.22 \\
\hline $10-11$ & 220.97 & 38.03 & - & - & - & 194.07 & 36.67 & 28.24 & - & 9.30 \\
\hline $12-13$ & 220.97 & 38.03 & - & - & - & 194.32 & 36.72 & 28.72 & - & 8.54 \\
\hline $13-14$ & 220.97 & 38.03 & - & - & - & 193.95 & 36.65 & 28.08 & - & 9.67 \\
\hline
\end{tabular}

Table 4: Critical loading margin for different scenarios

\begin{tabular}{ccccc}
\hline Line outage & Without Q & With Q & Line outage & With Q \\
\hline Base case & 2.236 & 2.276 & $5-6$ & 1.691 \\
$1-2$ & 0.881 & 1.191 & $6-11$ & 2.452 \\
$1-5$ & 2.350 & 2.245 & $6-12$ & 2.205 \\
$2-3$ & 1.489 & 1.610 & $6-13$ & 1.802 \\
$2-4$ & 1.774 & 1.851 & $7-9$ & 1.206 \\
$2-5$ & 1.813 & 1.869 & $9-10$ & 2.156 \\
$3-4$ & 1.956 & 1.959 & $9-14$ & 2.077 \\
$4-5$ & 2.365 & 2.309 & $10-11$ & 2.217 \\
$4-7$ & 2.045 & 2.011 & $12-13$ & 1.755 \\
$4-9$ & 2.185 & 2.083 & $13-14$ & 2.258 \\
\end{tabular}

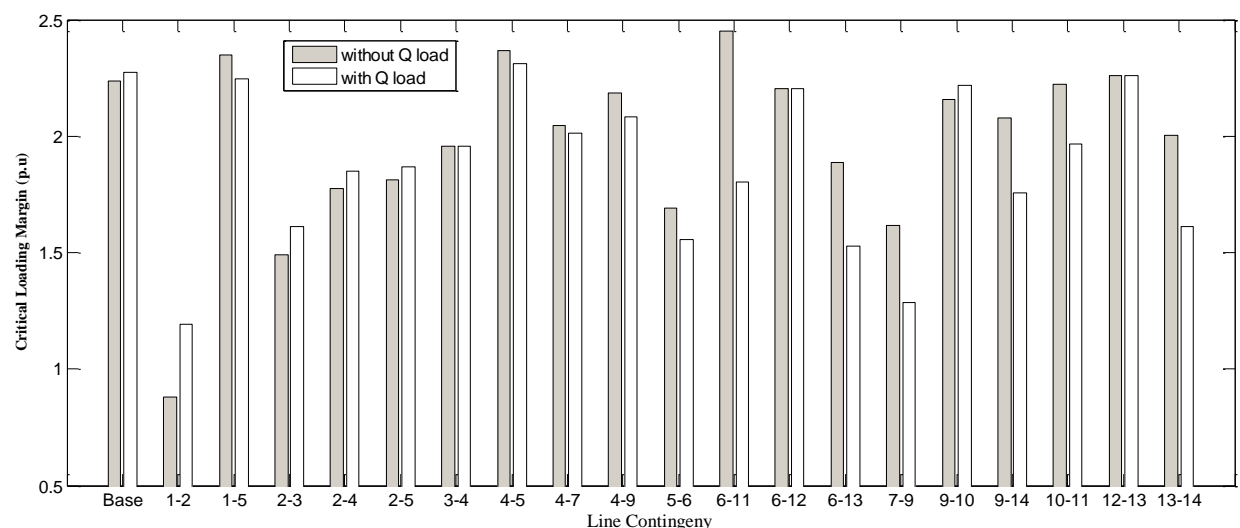

Fig 5: Impact of reactive power loading on CLM under various contingences

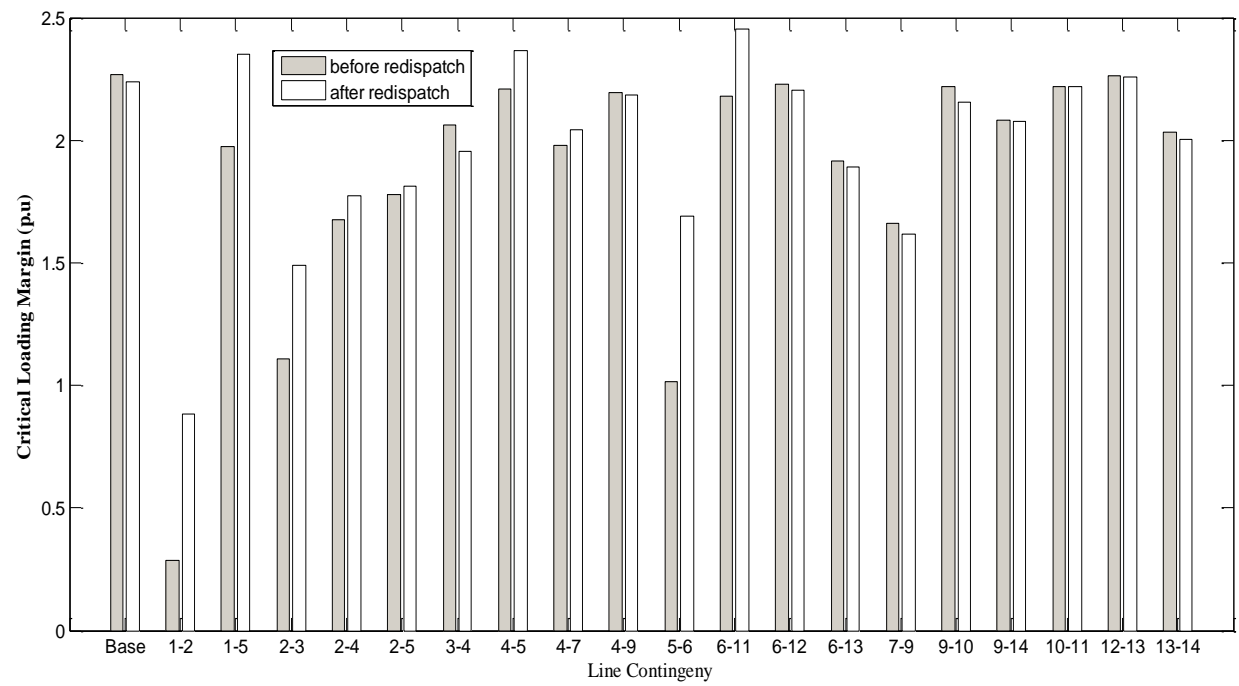

Fig 6: Impact of reschedule without VAr loading on CLM under various contingences 


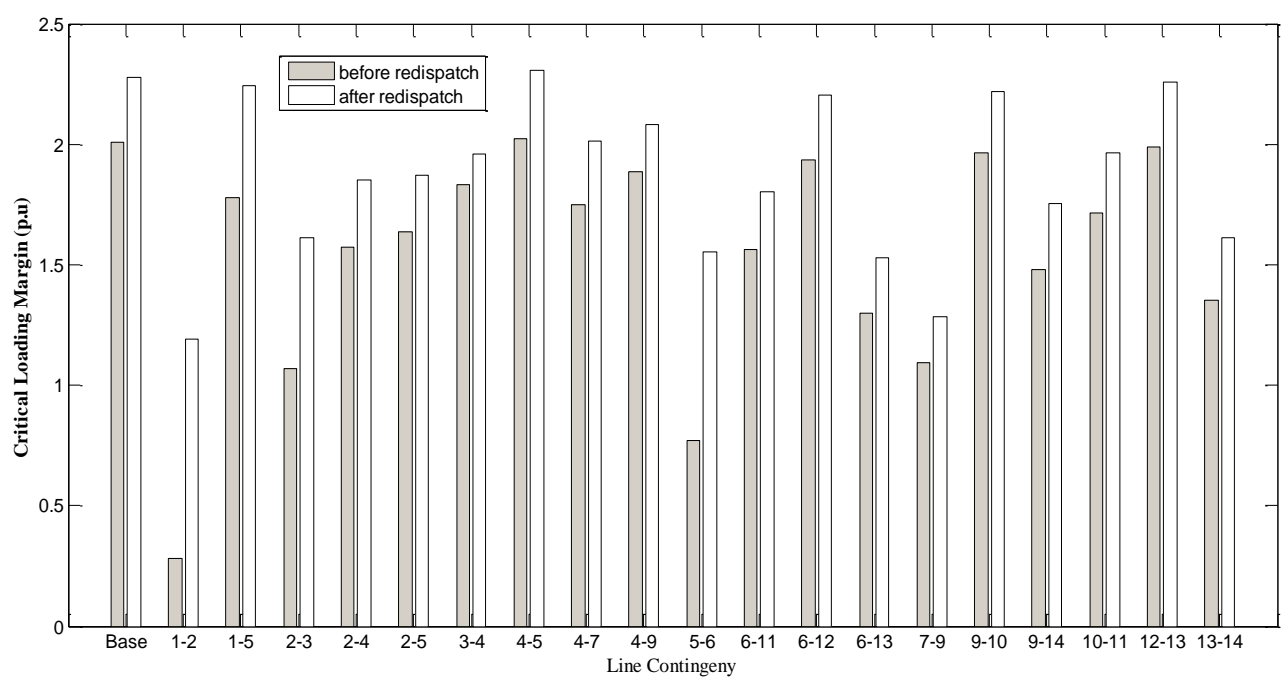

Fig 7: Impact of reschedule with VAr loading on CLM under various contingences

\section{Conclusions}

In this work, the reactive power loading effect on critical loading margin of the system is analyzed. In addition, the (N-1) line contingencies are also imposed to understand the transmission system support also to maximize CLM. From the case studies, the VAr loading as well as contingencies are caused to decrease the CLM considerably. In order to enhance the CLM, the transmission system security constrained reschedule is considered as a shortterm remedy. The results are well supporting the redispatch requirement under $(\mathrm{N}-1)$ line contingencies not only to ensure transmission system security but also to enhance critical loading margin. But the reschedule may cause for increase in operating cost and this problem has to overcome by strengthening the transmission system by using advanced technologies like FACTS devices. This will be the subject for the next work.

\section{References}

[1] P. Kundur, Power system stability and control, McGraw-Hill, Inc, 2007.

[2] Timothy J. E. Miller, Reactive Power Control in Electric Systems, Wiley- Blackwell, 1983.

[3] T. Gomez, J. Lumbreas, and V. Parra, "A Security-constrained Decomposition approach to optimal reactive power planning," IEEE Trans. on Power Systems, August 1991,Vol. 6, pp. 1069 1076,

[4] S. Graville and M. Lima, "Application of decomposition techniques to VAR planning: Methodological \& Computational Aspects," IEEE Trans. on Power Systems, Nov. 1994, Vol. 9, pp. 1780-1787.

[5] V. Ajjarapu, P.L. Lau, S. Battula, "An optimal reactive power planning strategy against voltage collapse", IEEE Trans. Power System. (1994) 9 (2) 906-917.

[6] E. Vaahedi et al., "Large scale voltage stability constrained Optimal VAR planning and voltage stability application using existing OPF/Optimal VAR Planning Tools", IEEE Trans. on Power Systems, Feb 1999, Vol. 14, pp. 65-74,.

[7] Kumar, Ashwani, S. C. Srivastava, and S. N. Singh. "Congestion management in competitive power market: a bibliographica survey," Electric Power Systems Research, 2005,Vol. 76, no. 1, pp. 153-164,

[8] Momoh, James A., and J. Z. Zhu. "Improved interior point method for OPF problems," IEEE Transactions on Power Systems, 1999,Vol. 14, no. 3, pp. 1114-1120,

[9] D.P.Kothari, Modern Power System Analysis,

[10] Ajjarapu, V.; Christy, C., "The continuation power flow: a tool for steady state voltage stability analysis," IEEE Transactions on Power Systems, Feb 1992, Vol.7, no.1, pp.416-423,.
[11] C.A. Canizares and F.L. Alvarado, "Point of collapse and continuation methods for large ac-dc systems", IEEE Transactions on Power Systems, Feb 1993,Vol. 8, no. 1, pp. 18 ,

[12] Amjady, Nima, and Masoud Esmaili, "Improving voltage security assessment and ranking vulnerable buses with consideration of power system limits," International journal of electrical power \& energy systems, 2003,Vol. 25 , no. 9 pp. 705 715 ,

[13] Ajjarapu, V.; Christy, C., "The continuation power flow: a tool for steady state voltage stability analysis," IEEE Transactions on Power Systems, Feb 1992, Vol.7, no.1, pp.416-423,

[14] Ruipeng, Guo, and Han Zhenxiang. "An improved continuation power flow method for voltage stability analysis," Automation of Electric Power Systems, 1999, Vol. 14,.

[15] Alves, Dilson, and Luiz Carlos Da Silva. "Study of alternative schemes for the parameterization step of the continuation power flow method based on physical parameters, part I: Mathematical modeling." Electric Power Components and Systems, 2003, Vol. 31, no. 12, pp. 1151-1166,

[16] R. D. Zimmerman, C. E. Murillo-Sanchez, and R. J. Thomas, "MATPOWER: Steady-State Operations, Planning and Analysis Tools for Power System Research and Education," IEEE Transactions on Power Systems, Feb. 2011, Vol. 26, no. 1, pp. 12-19. 